\title{
Enhanced surface diffusion through termination conversion during epitaxial $\mathrm{SrRuO}_{3}$ growth
}

\author{
Guus Rijnders ${ }^{\text {a) }}$ and Dave H. A. Blank \\ $\mathrm{MESA}^{+}$Institute for Nanotechnology, Inorganic Materials Science, Faculty of Science and Technology, \\ University of Twente, P.O. Box 217, 7500 AE Enschede, The Netherlands \\ Junghoon Choi and Chang-Beom Eom \\ Department of Materials Science and Engineering, University of Wisconsin-Madison, Madison, \\ Wisconsin 53706
}

(Received 14 October 2003; accepted 15 November 2003)

\begin{abstract}
During the initial growth of the ferromagnetic oxide $\mathrm{SrRuO}_{3}$ on $\mathrm{TiO}_{2}$-terminated $\mathrm{SrTiO}_{3}$, we observe a self-organized conversion of the terminating atomic layer from $\mathrm{RuO}_{2}$ to $\mathrm{SrO}$. This conversion induces an abrupt change in growth mode from layer by layer to growth by step advancement, indicating a large enhancement of the surface diffusivity. This growth mode enables the growth of single-crystalline and single-domain thin films. Both conversion and the resulting growth mode enable the control of the interface properties in heteroepitaxial multilayer structures on an atomic level. (C) 2004 American Institute of Physics. [DOI: 10.1063/1.1640472]
\end{abstract}

Complex oxides have attracted great interest for the wealth of different electrical and magnetic properties that these materials exhibit, such as ferromagnetism, antiferromagnetism, ferroelectricity, high dielectric constants, piezoelectricity, colossal magnetoresistivity, and high- $T_{c}$ superconductivity. Based on these properties, device concepts are under investigation, e.g., spin-polarized ferromagnetic tunnel junctions, superconducting electronics, and high-frequency piezoelectric transducers. One of the key issues in these complex oxide heterostructures is the short characteristic length scale at which the above-mentioned phenomena take place. Control of the growth and interface engineering on an atomic level are therefore prerequisites to utilize all opportunities.

$\mathrm{SrRuO}_{3}$ has been subject to many studies because of its unique structural, magnetic, and electronic properties, and chemical stability. ${ }^{1}$ The strong magnetocrystalline anisotropy in single-domain thin films ${ }^{2,3}$ as well as crystals makes $\mathrm{SrRuO}_{3}$ especially suitable for use in magnetic tunnel junctions, provided that single-domain heterostructures are achievable. ${ }^{4,5}$ Furthermore, ferromagnetic $\mathrm{SrRuO}_{3}$ has been used as a model system to study the initial growth, with special attention to surface termination and growth mode. ${ }^{6}$ Because their application is mostly in mirror-symmetric trilayer junction configurations, growth control of the individual layers, as well as the atomic stacking sequence at the interfaces, is required. This makes an in situ diagnostic tool indispensable. With the introduction of high-pressure reflection high-energy electron diffraction (RHEED), ${ }^{7}$ growth control with an atomic accuracy became possible during pulsed laser deposition (PLD) at pressures up to $100 \mathrm{~Pa}$.

Studying the homoepitaxial growth of $\mathrm{SrTiO}_{3}$ with highpressure RHEED, it became clear that the surface termination, from the substrate as well as the deposited film, is important to achieve well-defined growth. Only on $\mathrm{TiO}_{2}$-terminated $\mathrm{SrTiO}_{3}$ (Refs. 8 and 9) perfect layer-by-

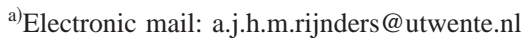

layer growth was obtained. ${ }^{10}$ In this study, vicinal $\mathrm{TiO}_{2}$-terminated $\mathrm{SrTiO}_{3}$ substrates have been used to study the initial growth of $\mathrm{SrRuO}_{3}$. PLD (KrF excimer laser; $\lambda=248 \mathrm{~nm}$ ) is used as deposition technique using a sintered $\mathrm{SrRuO}_{3}$ target. At the deposition conditions, about 19 pulses are needed for the deposition of one unit-cell layer. ${ }^{11}$

Figures 1(a)-1(c) show the intensity of the specular reflected RHEED spot during the growth of $\mathrm{SrRuO}_{3}$ at a deposition temperature of $600{ }^{\circ} \mathrm{C}$ on substrates with different vici-

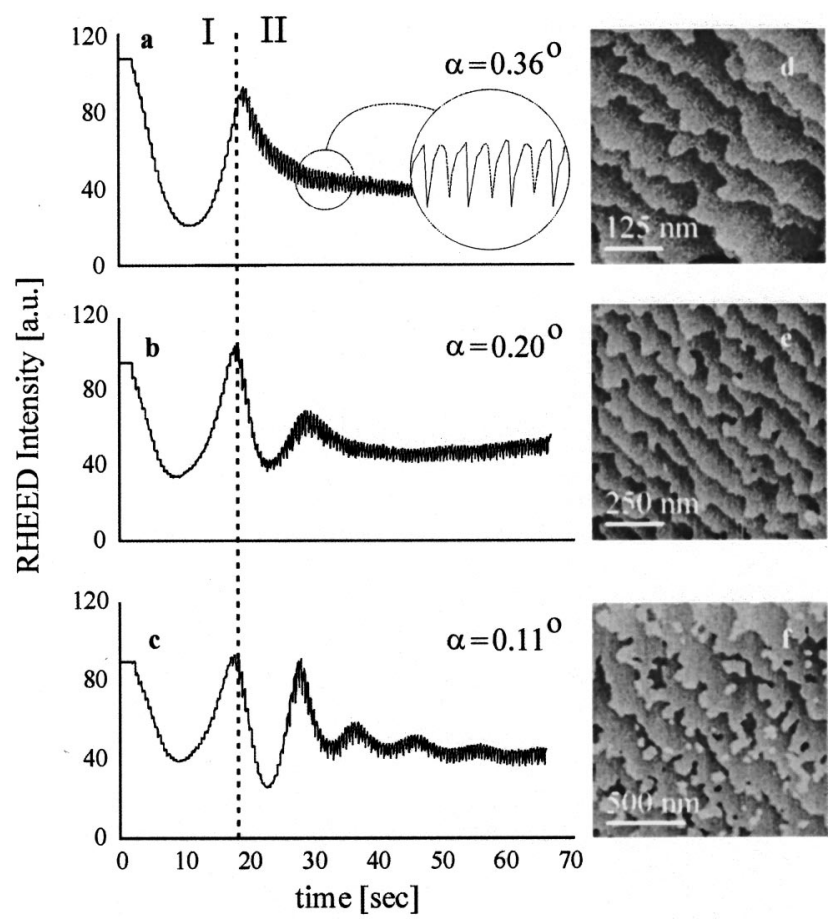

FIG. 1. RHEED intensity of $\mathrm{SrRuO}_{3}$ deposit at $600{ }^{\circ} \mathrm{C}$ on $\mathrm{TiO}_{2}$-terminated $\mathrm{SrTiO}_{3}$ substrates with different vicinal angles $\alpha$; (a) $\alpha=0.36^{\circ}$, (b) $\alpha=0.20^{\circ}$, (c) $\alpha=0.11^{\circ}$, and corresponding AFM images of $50 \mathrm{~nm} \mathrm{SrRuO}_{3}$ films using similar vicinal angles; (d) $\alpha=0.33^{\circ}$, (e) $\alpha=0.22^{\circ}$, (f) $\alpha=0.10^{\circ}$. The dotted line separates two different growth regimes; on the left-hand side (I), 2D layer by layer, on the right-hand side (II), mixed growth, i.e., layer by layer and step flow. Inset shows the RHEED intensity relaxations. 
nal angles, i.e., $0.36^{\circ}, 0.20^{\circ}$ and $0.11^{\circ}$, respectively. Two regimes with different growth modes are distinguishable. The oscillation in the first regime (marked I) indicates layerby-layer growth, and the number of pulses needed to complete the first RHEED oscillation is independent on the vicinal angle used. However, in the second regime (marked II), the intensity is strongly dependent on the vicinal angle. At the vicinal angle $\alpha$ of $0.36^{\circ}$, no additional oscillations are observed, whereas at smaller angles, multiple oscillations are observed. The number of oscillations increases with decreasing vicinal angle. However, in all cases, the oscillations fade away and a steady RHEED signal with high intensity is observed at the final growth stage, which indicates step-flowlike growth. Another feature in the RHEED intensity variation confirms the occurrence of step-flow growth. Because of the pulsed laser, the deposition is discontinuous; resulting in a modulated RHEED intensity. Initiated by the laser pulse, a very fast deposition of a small amount of material (in this case $\sim 5 \%$ of one unit-cell layer) takes place followed by a rearrangement of the deposit. This manifests as a relaxationlike behavior of the RHEED intensity, ${ }^{12,13}$ see inset of Fig. 1(a). If the step-flow growth is dominating, the amplitude of the RHEED intensity relaxation is large because of instantaneous deposition on an atomically smooth film surface. The atomic force microscope (AFM) images in Figs. 1(d)-1(f) show the surface morphologies of $50 \mathrm{~nm}$ thick $\mathrm{SrRuO}_{3}$ films on substrates with similar vicinal angles as used in Figs. 1(a) $-1(\mathrm{c})$, respectively. The morphology of the films clearly reflects the step-flow growth. At low vicinal angles, however, the probability for nucleation on the terraces is increased. As a result, islands are formed which explain the multiple oscillations observed at smaller vicinal angles. Coalescence of these islands with the advancing steps leads to meandering step ledges. This process of nucleation of islands between flowing step ledges, and the coalescence of the islands with the flowing ledges, will take place continuously resulting in a constant step density and, therefore, a steady RHEED intensity. In the AFM images, this steady state can be seen: The average distance between the step edges of both islands and terraces of low vicinal substrates becomes of the same order as the high vicinal one. This mixed growth is theoretically predicted before ${ }^{14}$ and, here, experimentally observed in a complex oxide system.

The transition in growth mode, i.e., layer by layer in regime I and step-flowlike in regime II, implies a change in the surface diffusivity during the initial growth of $\mathrm{SrRuO}_{3}$ on $\mathrm{TiO}_{2}$-terminated $\mathrm{SrTiO}_{3}$. To elucidate this transition, the growth behavior in regime I is studied in more detail.

Figure 2(a) shows the RHEED intensity recorded at growth temperatures of 500,600 , and $700{ }^{\circ} \mathrm{C}$ on $0.11^{\circ}$ vicinal angle substrates. During the initial growth, the oscillation periods are not equal in time. Specifically, the number of pulses to complete the first oscillation is large compared to the expected 19 pulses needed to complete one unit-cell layer, and depends heavily on the deposition temperature, i.e., $\sim 26,32$, and 37 for 500,600 , and $700^{\circ} \mathrm{C}$, respectively. At $700{ }^{\circ} \mathrm{C}$, the number of pulses becomes almost equal to the number of pulses needed for depositing two unit-cell layers, while the period of the subsequent oscillations is equivalent to the expected number of pulses. The origin of this delay in Downloaded 01 Mar 2007 to 128.104.198.190. Redistribution subject

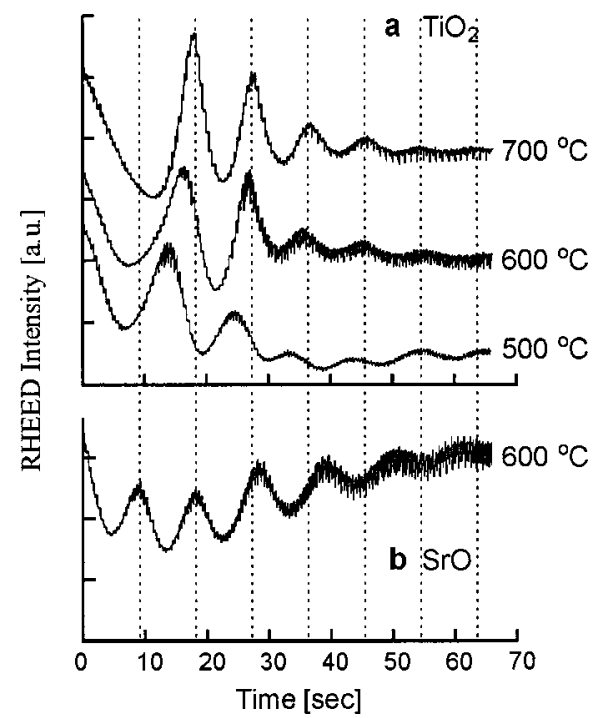

FIG. 2. RHEED intensity at different deposition temperatures on vicinal $\left(\alpha=0.11^{\circ}\right) \mathrm{TiO}_{2}$-terminated $\mathrm{SrTiO}_{3}$ substrates (a). The dotted lines indicate the time where completion of one unit-cell layer is expected at $700{ }^{\circ} \mathrm{C}$ and should be used as a guide for the eye. (b) RHEED intensity during the growth of $\mathrm{SrRuO}_{3}$ on $\mathrm{SrO}$-terminated $\mathrm{SrTiO}_{3}$.

RHEED intensity cannot be explained by a phase shift since the diffraction conditions are kept constant during all experiments. Since the first oscillation period is independent on the vicinal angles used, the delay also cannot be explained by a mixed growth mode of $2 \mathrm{D}$ layer by layer and step flow. ${ }^{15}$ This is supported by the very asymmetric curvature of the first RHEED oscillation. A minimum intensity is reached after approximately 27 pulses, equivalent to nearly the deposition of 1.5 unit-cell layers. Figure 3(a) shows an AFM micrograph interrupting the growth at this minimum. Many small islands can be observed. The height of these islands is about $4 \AA$ (at room temperature, the $c$-axis lattice parameter of the $\mathrm{SrRuO}_{3}$ pseudo-cubic cell is $3.93 \AA$ ). Figure 3(b) shows the surface morphology at the first RHEED maximum. As expected from the RHEED data, an almost complete layer is observed. During this initial growth, the stacking sequence of the perovskite structure at the substrate-film interface is preserved, as determined by high-resolution electron microscopy analysis. ${ }^{16}$

The observed longer asymmetric first oscillation, the perovskite stacking sequence, as well as the surface morphology at minimum RHEED intensity can be explained by one phenomenon: A termination conversion from the B site
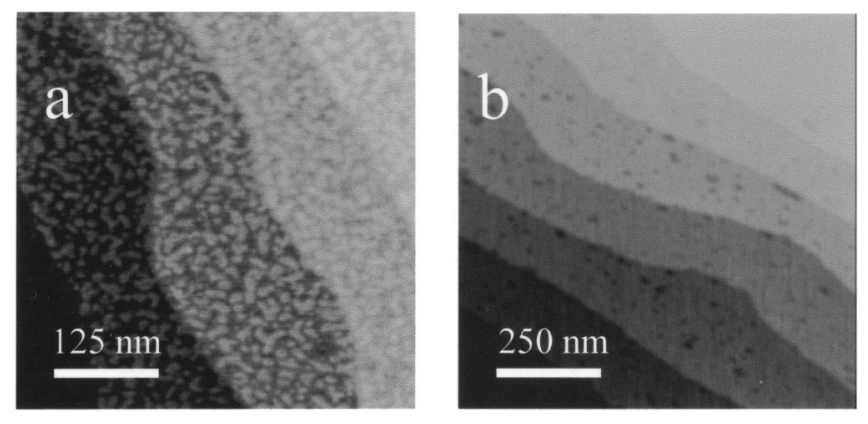

FIG. 3. AFM images of $\mathrm{SrRuO}_{3}$ on $\mathrm{TiO}_{2}$-terminated $\mathrm{SrTiO}_{3}$ taken at the first minimum (a) and first maximum (b) of the RHEED intensity as presented in Fig. 2(a) at $700{ }^{\circ} \mathrm{C}$.

to AIP license or copyright, see http://apl.aip.org/apl/copyright.jsp 


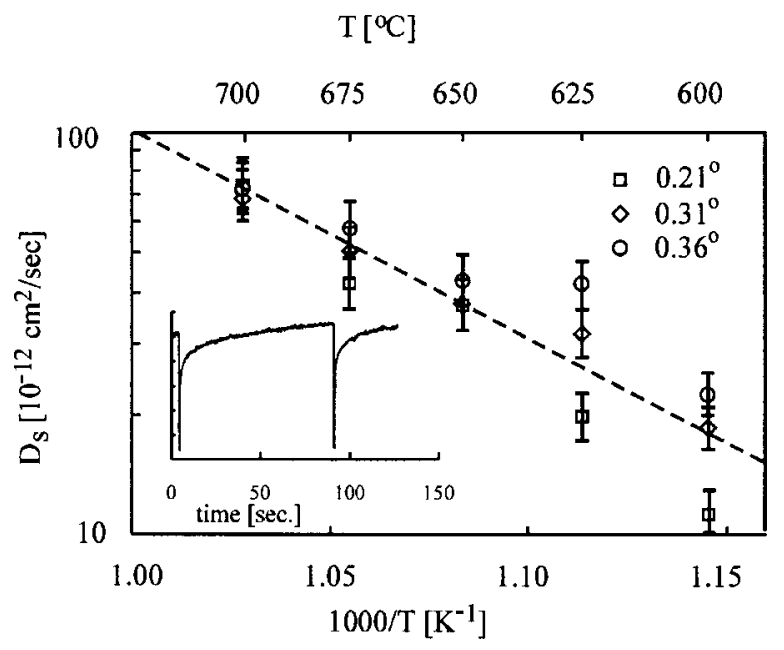

FIG. 4. The diffusivity, estimated from the recovery time of RHEED intensity for different vicinal angles and temperatures in the Arrhenius form. The dotted line is a guide for the eyes. The inset shows a typical example of RHEED intensity recovery between deposition pulses at $675^{\circ} \mathrm{C}$.

$\left(\mathrm{TiO}_{2}\right.$-terminated $\left.\mathrm{SrTiO}_{3}\right)$ to A site (SrO-terminated $\mathrm{SrRuO}_{3}$ ) in the first unit-cell layer. This is opposite to what one would expect: Preservation of perovskite stacking and stoichiometric deposition would lead to $\mathrm{RuO}_{2}$ termination. This latter termination does not occur, apparently because it is not stabilized at these deposition conditions. Therefore, $\mathrm{SrRuO}_{3}$ decomposes to $\mathrm{SrO}$ and highly volatile $\mathrm{Ru}_{x} \mathrm{O}_{y}$. This decomposition stops after the terminating layer is completely converted to $\mathrm{SrO}$. At lower temperatures, this conversion is distributed over several oscillation periods, whereas at $700{ }^{\circ} \mathrm{C}$, it is completed within the first unit-cell layer.

In the case of $\mathrm{SrO}$-terminated ${ }^{16} \mathrm{SrTiO}_{3}$ substrates ( $a$-side termination), one would not expect this conversion. As can be seen in Fig. 2(b), the period of the first RHEED intensity oscillation is exact the amount material to complete one unit-cell layer. The perovskite sequence is maintained and the evaporation of $\mathrm{RuO}$ and the accompanying termination conversion at the initial growth stage of $\mathrm{SrRuO}_{3}$ do not occur.

After the conversion in termination is complete, an enhancement of the surface diffusivity is observed, leading to a change in growth mode from layer by layer toward step flow, as shown in Fig. 1. As mentioned before, the rearrangement of deposited material leads to RHEED intensity relaxations between deposition pulses. At step-flow conditions, no nucleation takes place and the average step density, determined by the vicinal angle, is constant. The recovery of the specular RHEED intensity is therefore a direct measure for the decay of adatom density, and thus adatom diffusivity, between the deposition pulses. A typical example of RHEED intensity recovery is shown in the inset of Fig. 4 . The diffusivity $D_{S}$, parameterized by the activation energy for diffusion $E_{A}$, $D_{S} \sim \exp \left(-E_{A} / k_{B} T\right)$, can be estimated from the terrace width $\ell_{T}$ and the decay time of the adatom density on the terraces determined from the RHEED intensity recovery. ${ }^{17}$ Figure 4 shows the diffusivity $D_{S}$ versus the inverse temperature for three different vicinal angles, i.e., $0.21^{\circ}, 0.31^{\circ}$, and $0.36^{\circ}$, resulting in three different terrace widths, i.e., 110, 74, and $64 \mathrm{~nm}$, respectively. From Fig. 4, a value of $1.0 \pm 0.2 \mathrm{eV}$ is found for $E_{A}$. This value is relatively low compared to values found for other complex oxides, like $\mathrm{SrTiO}_{3}$ (Ref. 15) and $\mathrm{YBa}_{2} \mathrm{Cu}_{3} \mathrm{O}_{7-x},{ }^{18}$ i.e., $2-4 \mathrm{eV}$ and $2-2.6 \mathrm{eV}$, respectively. Because of the higher values for $E_{A}$, PLD of the latter two complex oxides at comparable deposition conditions using similar vicinal substrates typically results in 2D growth. Reliable determination of $E_{A}$ at the initial 2D growth stage of $\mathrm{SrRuO}_{3}$ is not possible, since both the nucleation density and termination conversion are temperature dependent. As a result, the average distance between surface steps, i.e., initial substrate steps and island ledges, is not constant.

Conductive ferromagnetic oxide $\mathrm{SrRuO}_{3}$ has been grown on $\mathrm{TiO}_{2}$-terminated $\mathrm{SrTiO}_{3}$. It is demonstrated that during the initial growth the termination converts from the $\mathrm{B}$ to the A site by the following observations: An exact double RHEED oscillation period at high growth temperature on $\mathrm{TiO}_{2}$-terminated substrates, whereas no doubling is observed with the $\mathrm{SrO}$ termination; The period of the first oscillation is independent on the vicinal angle of the substrates; The first oscillation has an asymmetric shape; the first minimum shifts toward 1.5 unit-cell deposition; An abrupt change in growth mode just after completing the first (double) oscillation period; Only single-unit-cell steps of approximately $4 \AA$ at the first RHEED intensity minimum.

The diffusivity is enhanced after this conversion resulting in step-flowlike growth, which is a mixture of 2D growth and growth by step advancement and depends on the terrace width. This study shows that the manipulation of the terminating atomic layer and, consequently, the interface in heteroepitaxial structures is feasible, resulting in the possibility to grow mirror-symmetric junctions on an atomic level.

${ }^{1}$ C. B. Eom, R. B. Vandover, J. M. Phillips, D. J. Werder, J. H. MarshallL, C. H. Chen, R. J. Cava, R. M. Fleming, and D. K. Fork, Appl. Phys. Lett. 63, 2570 (1993).

${ }^{2}$ L. Klein, J. S. Dodge, C. H. Ahn, G. J. Snyder, T. H. Geballe, M. R. Beasley, and A. Kapitulnik, Phys. Rev. Lett. 77, 2774 (1996).

${ }^{3}$ Q. Gan, R. A. Rao, C. B. Eom, L. Wu, and F. Tsui, J. Appl. Phys. 85, 5297 (1999).

${ }^{4}$ C. B. Eom, R. J. Cava, R. M. Fleming, J. M. Phillips, R. B. Vandover, J. H. Marshall, J. W. P. Hsu, J. J. Krajewski, and W. F. Peck, Science 258, 1766 (1992).

${ }^{5}$ Q. Gan, R. A. Rao, and C. B. Eom, Appl. Phys. Lett. 70, 1962 (1997).

${ }^{6}$ J.-H. Choi, C. B. Eom, G. Rijnders, H. Rogalla, and D. H. A. Blank, Appl. Phys. Lett. 79, 1447 (2001).

${ }^{7}$ G. J. H. M. Rijnders, G. Koster, D. H. A. Blank, and H. Rogalla, Appl. Phys. Lett. 70, 1888 (1997)

${ }^{8}$ G. Koster, B. L. Kropman, G. J. H. M. Rijnders, D. H. A. Blank, and H. Rogalla, Appl. Phys. Lett. 73, 2920 (1998).

${ }^{9}$ M. Kawasaki, K. Takahash, T. Meada, R. Tsuchiya, M. Shinhara, O. Ishiyama, T. Yonezawa, M. Yoshimoto, and H. Koinuma, Science 226, 1540 (1994).

${ }^{10}$ S. Stoyanov, Surf. Sci. 199, 226 (1988).

${ }^{11}$ The average growth rate was approximately $0.2 \AA$ Apulse leading to 19 laser pulses to deposit one pseudocubic unit cell layer.

${ }^{12}$ H. Karl and B. Stritzker, Phys. Rev. Lett. 69, 2939 (1992).

${ }^{13}$ D. H. A. Blank, G. J. H. M. Rijnders, G. Koster, and H. Rogalla, Appl. Surf. Sci. 129, 633 (1998).

${ }^{14}$ Z. S. Yanovitskaya, I. G. Neizvestny, N. L. Shwartz, M. I. Katkov, and I. P. Ryzhenkov, Appl. Surf. Sci. 123, 729 (1998).

${ }^{15}$ G. Rijnders, Ph.D. thesis, University of Twente, ISBN 90-365-1657-9, 2001

${ }^{16}$ G. Rijnders, G. Koster, V. Leca, D. H. A. Blank, and H. Rogalla, Appl. Surf. Sci. 168, 223 (2000).

${ }^{17}$ X. D. Zhu, Phys. Rev. B 58, 10975 (1998).

${ }^{18}$ B. Dam and B. Stauble-Pumpin, J. Mater. Sci.: Mater. Electron. 9, 217 (1998). 